\title{
Recurrent tracheal tumor with a critical airway requiring ' $Y$ ' stent - unique presentation of tuberculosis
}

\author{
Venugopal Jaganathan, Santhakumar Subramanian, Deepak T Hari \\ Department of interventional Pulmonology, Kovai Medical Center and Hospital, Coimbatore, India
}

\begin{abstract}
Tracheal tumor is a rare entity. Tracheal tumor may be a primary tracheal tumor or secondary to invasion from a mediastinal tumor (or a lymph node). These tumors are prone to cause critical airway obstruction which may require urgent care. Tuberculosis is one of the common differential diagnoses of mediastinal lymphadenopathy in TB endemic countries, though isolated tuberculous mediastinal lymphadenopathy without a lung involvement is rare. We report an extremely rare case of isolated paratracheal lymphadenitis due to tuberculosis, eroding the trachea and presented a lower tracheal tumor, which recurred again after complete debulking. Finally, the disease required a ' $\mathrm{Y}$ ' stent placement, to stabilize the airway following the second recanalization. We discuss the incidence, differential diagnosis, and the bronchoscopic, interventional aspects of this entity.
\end{abstract}

Correspondence: Santhakumar Subramanian, Consultant Interventional Pulmonologist, Kovai Medical Center and Hospital, 99 Avinashi road, Coimbatore 14, India.

Tel. +91.9659163000 .

E-mail: drshansharan@gmail.com

Contributions: All the authors made a substantive intellectual contribution. All the authors have read and approved the final version of the manuscript and agreed to be accountable for all aspects of the work.

Conflict of interest: the authors declare no conflict of interest. Informed consent: Appropriate written informed consent was obtained for publication of this case series and accompanying images.

Key words: Paratracheal mass; mediastinal lymphadenopathy; stridor; rigid bronchoscopy; recanalization.

Received for publication: 20 August 2020.

Accepted for publication: 1 January 2021.

${ }^{\circ}$ Copyright: the Author(s), 2021

Licensee PAGEPress, Italy

Monaldi Archives for Chest Disease 2021; 91:1578

doi: 10.4081/monaldi.2021.1578

This article is distributed under the terms of the Creative Commons Attribution Noncommercial License (by-nc 4.0) which permits any noncommercial use, distribution, and reproduction in any medium, provided the original author(s) and source are credited.

\section{Introduction}

Tracheal tumors represent about $0.2 \%$ of the tumors of the respiratory system. In general, most of the tracheal tumors are malignant. The benign tracheal tumors including inflammatory polyps, chondroma, granular cell tumors, and neurogenic tumors are rare [1]. Tracheal tumors may be classified as, either primary tracheal tumors or secondary to invasion from nearby structures. Paratracheal metastatic lymph nodes and malignant thyroid lesions are the common, among those invade the trachea [2]. However, tuberculosis (TB) has to be considered in the differential diagnosis, in TB endemic countries [3] like India, though isolated mediastinal lymphadenitis without a lung involvement in TB is rarely reported in immunocompetent people [4]. Mediastinal tumors cause respiratory symptoms, usually, due to compression of the airway as they enlarge, but an erosion of tracheal wall and direct invasion leading to a critical airway obstruction may occur, which requires an intervention at the earliest [5]. We report a rare case of an immunocompetent middle-age female, presented with stridor, who had a lower tracheal tumor with a critical airway obstruction. She was diagnosed to have a tuberculous paratracheal lymphadenitis invading the trachea. Unfortunately, the tumor recurred again after complete debulking, despite a proper anti-TB chemotherapy. The airway was recanalized again followed by a ' $Y$ ' stent placement, to stabilize the airway and prevent a recurrence, until treatment is completed.

\section{Case Report}

A forty years old female, presented with cough, exertional dyspnea, and noisy respiration for 3 weeks duration. She was a nondiabetic female, without any other comorbidities. On examination, she had a stridor, predominantly during inspiration. Her respiratory rate was $22 / \mathrm{min}$, heart rate was $104 / \mathrm{min}$, and peripheral oxygen saturation was $92 \%$ at room air. She had no palpable peripheral lymphadenopathy and her systemic examination was unremarkable. Her blood biochemistry (urea, creatinine, complete blood count, random blood sugar) were within normal limits. Chest radiography showed a right-sided paratracheal mass, protruding into the trachea and narrowing the lumen. Contrast-enhanced computed tomography (CECT) scan of the chest showed a large right paratracheal homogenous mass, invading the lower trachea, just above the Carina, causing $70-80 \%$ of luminal obstruction (Figure 1). Flexible bronchoscopy (BF-1T180 Video Bronchoscope, Olympus, Tokyo, Japan) showed a mass lesion from the anterolateral wall of the lower trachea with a broad base which extended to the right main bronchus. The tumor had a smooth surface covered by a whitish slough like material and, it occluded almost $80 \%$ of the tracheal 
lumen. A provisional diagnosis of a malignant paratracheal tumor with tracheal invasion was made. Subsequently a rigid bronchoscopic (KARL STORZ Endoscopy-America) debulking of the tumor (Figure 2) was done, using an electrocautery knife (Reusable electrosurgical knife - KD-31C-1, Olympus) for cutting, an argon plasma coagulation (APC) (APC probe, $2.3 \mathrm{~mm}$, flexible, length $2.2 \mathrm{~m}$, ERBE, Tubingen, Germany) for hemostasis and a cryoprobe (ERBE, Tubingen, Germany; diameter $2.4 \mathrm{~mm}$, length $780 \mathrm{~mm}$ ) for extraction of the tumor. Adequate cryoprobe biopsies were done before debulking. The tumor could not be snared in total, due to its broad base. Biopsied specimens were subjected to histopathological and microbiological analysis. Histopathology showed necrotizing granulomatous inflammation. Acid Fast staining was negative but the GeneXpert -RIF/MTB test was positive and showed a rifampicin sensitivity. Hence, she was started on anti-tuberculous treatment (ATT) with a standard four drugs regimen (Rifampicin, Isoniazid, Ethambutol, Pyrazinamide) and discharged as she was asymptomatic. After 4 weeks of symptom-free period, unfortunately, she returned with stridor again and, a repeat imaging followed by a bronchoscopy showed a recurrent tumor at the same place. In view of her critical symptoms, she was subjected to a rigid bronchoscopic recanalization again, followed by a silicon ' $\mathrm{Y}$ ' stent placement to prevent further recurrence and occlusion of the airway (Figure 2). ' $\mathrm{Y}$ ' stent was chosen because, the disease, particularly the base of the tumor had an extension to the right main bronchus. She was advised to undergo a mediastinoscopy and biopsy of the paratracheal part of the tumor to rule out an underlying malignancy, because of the tumor recurrence despite ATT. But she refused to undergo any further invasive procedure. Histopathology of the recurrent tumor showed necrotizing granulomatous inflammation. There was no evidence of any malignancy or lymphoma. AFB- culture from both the primary and recurrent disease were negative after six weeks. She was continued on ATT. Within next eight weeks, on imaging, the paratracheal almost 50\% reduction in size. She had completed the full course of ATT in six months and a follow-up imaging revealed a near-total resolution of the paratracheal lesion. The stent was removed after four weeks of completion ATT (Figure 3). On one-year follow up after stent removal, she was asymptomatic and her chest radiology was normal.

\section{Discussion}

This case is a perfect example of a rare presentation of a common disease. Common tumors of the anterior superior mediastinum include thymoma, lymphoma, teratoma, thyroid mass, vascular lesions, and metastatic or granulomatous lymph nodes. Though TB is common in developing countries, an isolated paratracheal mass with a tracheal invasion is rare in tuberculosis [6], [7]. Due to, an indolent course of the disease, non-specific symptomatology, a normal chest radiography at early stages, and a low index of suspicion, it is not uncommon that, tracheal tumors are misdiagnosed and treated as asthma at early stages. They present in later stages with stridor [8] and critical airway obstruction. Contrast-enhanced CT scan helps
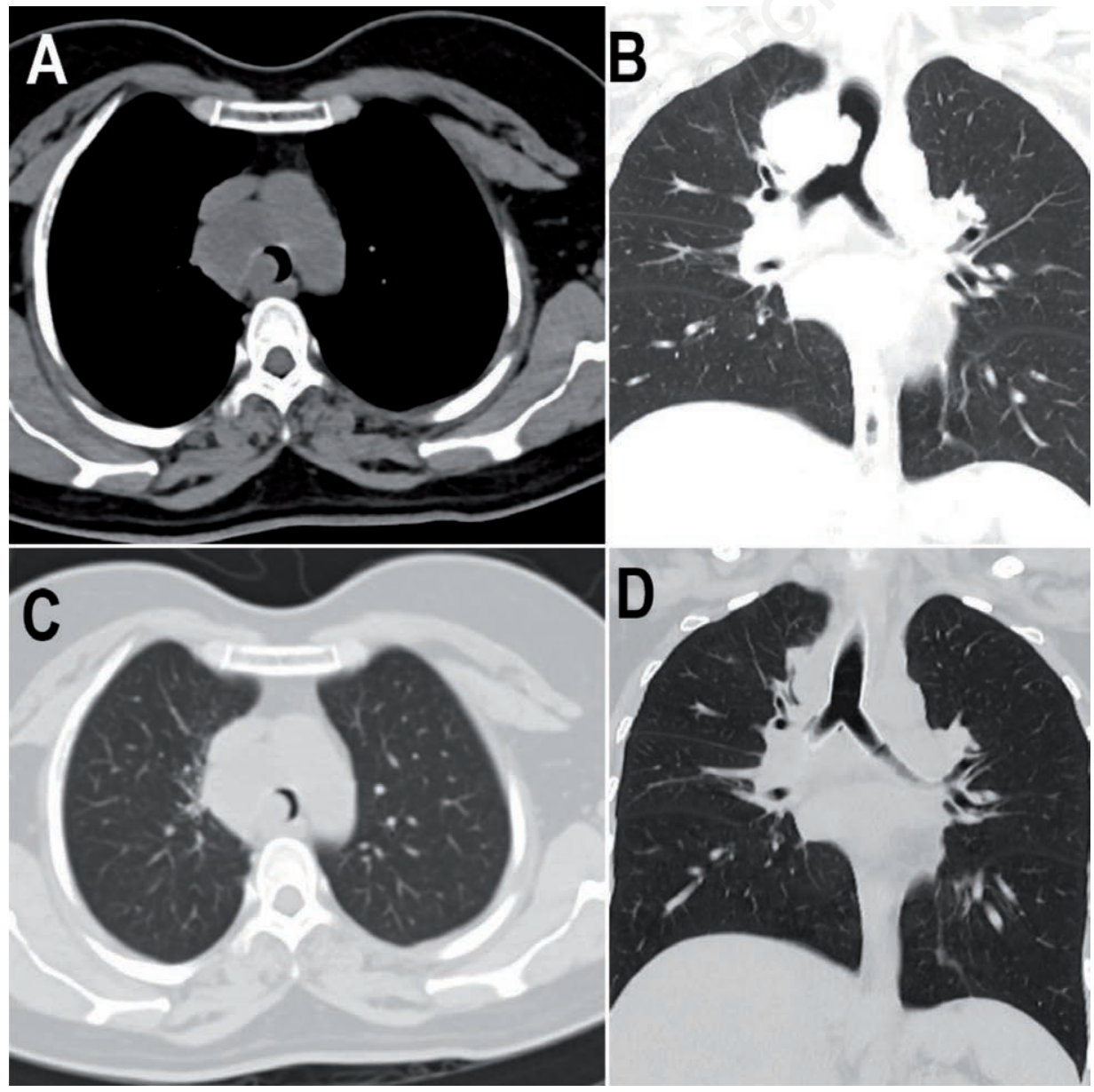

Figure 1. CT scan of lungs. Tumor shown in axial view: A) Mediastinal window; B) tumor seen in sagittal view with broad base extending just in to right main bronchus; $\mathrm{C}$ ) lung window; D) image after ' $Y$ ' stent placement post debulking of recurrent tumor. 
in narrowing the differential diagnosis, based on, the location, vascularity, amount of necrosis, matted or discrete lesions, ring-like enhancement, and involvement of nearby structures [9]. However, a tissue diagnosis is warranted always.

Bronchoscopy gives more accurate information about, the luminal compromise, surface, shape, and extension of the tumor. A flexible bronchoscopy may be used for an initial survey of the airway. Rigid bronchoscopy is preferred for any further intervention because, it is superior to flexible bronchoscopy in securing the airway with simultaneous ventilation, especially, in a situation of a sudden loss of the airway due to bleeding or edema during handling of the tumor for biopsy or debulking [5]. Management of tracheal tumor consists of, an emergent initial airway recanalization and a following definitive treatment (either surgical of palliative), which may be planned according to the pathological diagnosis.

Tuberculosis is one among the most common infectious diseases, kills nearly 1.4 million people every year worldwide [10]. Lymphadenitis is the second most common form of tuberculosis. Six months standard first-line regimen (2RHZE/4RHE) is recommended for lymph node TB as per the Indian guidelines [11]. This patient is unique because, the tumor recurred within a month after debulking, though the patient was treated with a proper ATT. The early recurrence challenged the first diagnosis and, raised a suspicion of a multi-drug resistant TB (MDR -TB) or a different pathology such as, lymphoma or carcinoma [12]. Hence, this patient was advised to undergo mediastinoscopy and biopsy of the extraluminal part of the tumor to confirm the first diagnosis. In India, any drug resistance is $22.54 \%$ among new patients and, $36.82 \%$ among previously treated patients. MDR-TB is reported in $2.84 \%$ among new patients and, $11.6 \%$ among previously treated patients [13].

Placement of an airway stent after recanalization is usually required, particularly if the airway is unstable due to malacia or an extensively damaged cartilage. At times, stenting is required as a bridge to a later surgical or another form of definitive treatment. Silicon stents are preferred over metallic self-expandable stents in benign central airway obstruction because, silicon stents are, less prone to cause a granulation reaction, customizable in different sizes and, easily repositioned or removed. We believe that the placement of the silicon ' $Y$ ' stent and not a straight one in the current patient is justified because, a straight tracheal stent, if placed in lower trachea near Carina, is more migrant and, does not cover the tumor base (which had an extension to the right main bronchus in this case). However, deployment of a ' $\mathrm{Y}$ ' stent is technically more challenging $[14,15]$ than a straight stent. To the best of our knowledge, this is the first case in the literature, where tuberculosis presented as a recurrent tracheal tumor masquerading as malignancy and, managed with a placement of a ' $\mathrm{Y}$ ' stent.

\section{Conclusions}

We conclude that, tracheal tumors are notorious to cause significant airway obstruction requiring emergency bronchoscopic
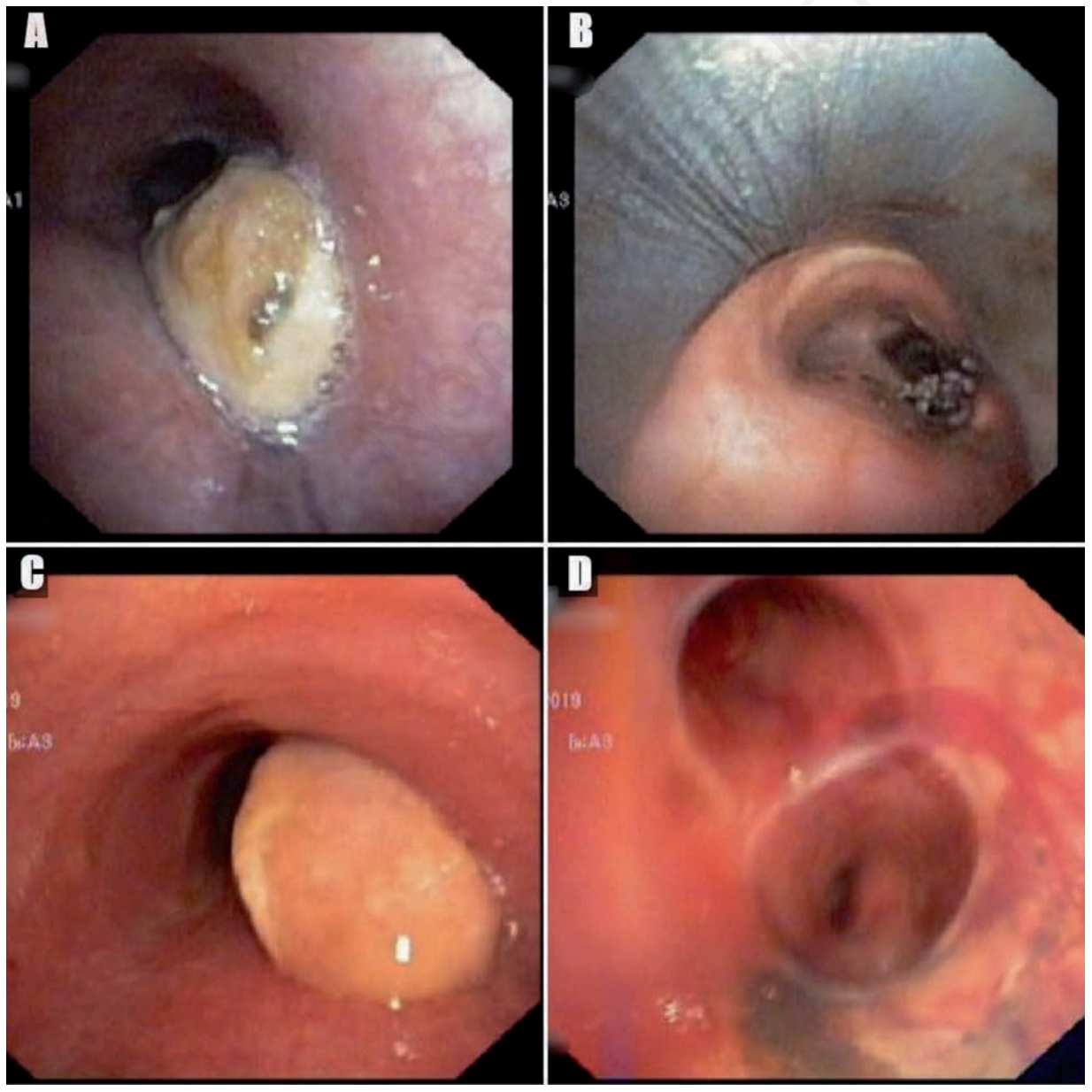

Figure 2. Bronchoscopic images. A) The mass lesion in lower trachea; B) post debulking; C) recurrent tumor; D) post ' $Y$ ' stent placement. 


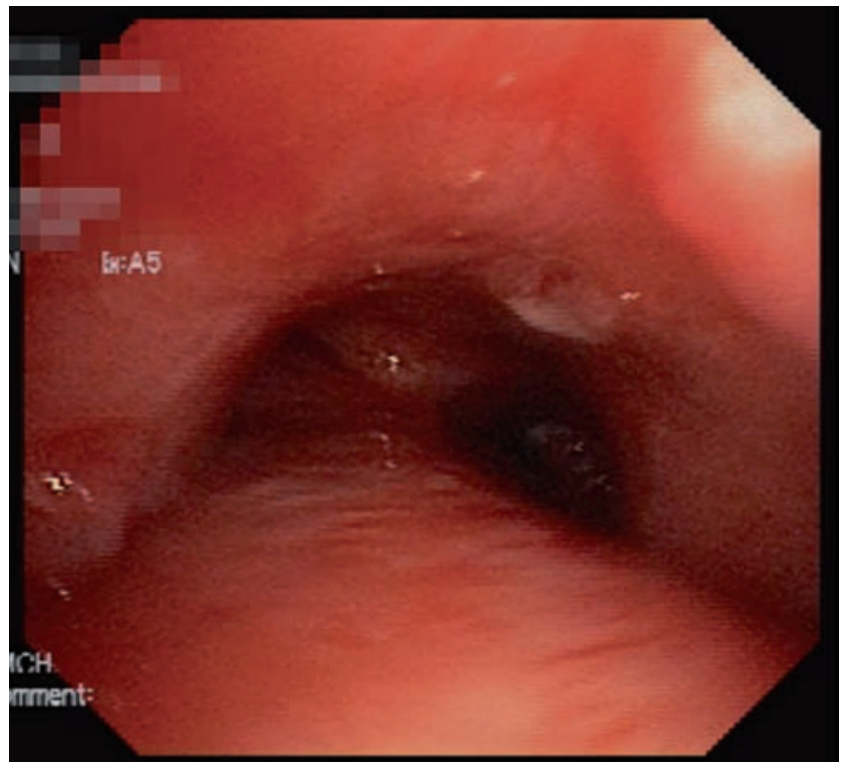

Figure 3. Bronchoscopy image of lower trachea after 6 months of stent removal, showing no recurrence.

intervention. Tuberculous mediastinal lymph node that erodes trachea and presents as a masquerade of malignancy is rare but exists. A high index of suspicion with a correlation of clinical, radiological, and pathological data, together with a prompt bronchoscopy, is required for early diagnosis and proper treatment.

\section{References}

1. Diaz-Mendoza J, Debiane L, Peralta AR, Simoff M. Tracheal tumors. Curr Opin Pulm Med 2019;25:336-43.
2. Madariaga ML, Gaissert HA. Secondary tracheal tumors: a systematic review. Ann Cardiothorac Surg 2018;7:183-96.

3. Gupta P. Difficulties in managing lymph node tuberculosis. Lung India 2004;21:50-3.

4. Khilnani GC, Jain N, Hadda V, Arava SK. Anterior mediastinal mass: A rare presentation of tuberculosis. J Trop Med 2011;2011:1-4.

5. Yildirim E. Principles of urgent management of acute airway obstruction. Thorac Surg Clin 2018;28:415-28.

6. Aroor AR, Rama Prakasha S, Seshadri S, et al. A study of clinical characteristics of mediastinal mass. J Clin Diagnostic Res 2014;8:77-80.

7. Hochhegger B, Zanetti G, Marchiori E. Mass invading the trachea: a rare presentation of tuberculosis simulating lung cancer. Infection 2013;41:599-600.

8. Seif F, Montenegro H. An enlarging solitary mass in an asymptomatic patient. Am J Med 2011;124:611-3.

9. Juanpere S, Cañete N, Ortuño P. A diagnostic approach to the mediastinal masses. Insights Imaging 2013;4:29-52.

10. Mondoni M, Repossi A, Carlucci P, et al. Bronchoscopic techniques in the management of patients with tuberculosis. Int $\mathrm{J}$ Infect Dis 2017;64:27-37.

11. World Health Organization. Country Office for India. IndexTB guidelines: Guidelines on extra-pulmonary tuberculosis for India. Central TB Division; Ministry of Health and Family Welfare, Government of India. 2016. Available from: https://apps.who.int/iris/handle/10665/278953

12. Pawlewicz K, Szutkowski Z, Kawecki A. Recurrence of adenoid cystic carcinoma of the trachea treated with radical radiotherapy: A case report. Oncol Lett 2018;15:3890-4.

13. Central TB Division, Ministry of Health \& Family Welfare, Government of India. Guideline for PMDT in India 2017. Available from: https://tbcindia.gov.in/index1.php?lang=1\& level $=2 \&$ sublinkid $=4780 \&$ lid $=3306$

14. Lee P, Kupeli E, Mehta AC. Airway Stents. Clin Chest Med 2010;31:141-50.

15. Folch E, Keyes C. Airway stents. Ann Cardiothorac Surg 2018;7:273-83. 